\title{
EL CONCEPTO DE SITUS OCUPACIONAL: POR QUÉ ESTA PROPUESTA NO ES PLAUSIBLE. UNA EXPLICACIÓN SOCIOLÓGICA
}

\author{
THE CONCEPT OF OCCUPATIONAL SITUS: WHY THIS NOTION IS NOT \\ PLAUSIBLE. A SOCIOLOGICAL EXPLANATION
}

\author{
RicARDO A. AyALA
}

\begin{abstract}
RESUMEN
Seis años atrás apareció en este mismo medio una propuesta desde el interior de la enfermería académica, que sugería el desarrollo -y aun la preexistencia- de un situs ocupacional de enfermería. Siguiendo este concepto, la enfermería podría tomar distancia de la dominación médica e instalar un situs propio de enfermería, con las enfermeras en la cúspide de la estructura y otras ocupaciones asociadas en posiciones subalternas. Esta proposición, aunque muy prometedora, no logró despertar mayor discusión en las revistas del rubro. En parte, esta omisión puede ser resultado de que al tratarse de un concepto de limitado interés empírico, aun para la sociología de las profesiones (desde donde proviene), el situs ocupacional se ha enmohecido en el olvido. En el presente artículo tendré como objetivo el argumentar por qué los conceptos de situs y situs ocupacional, además de tener poca utilidad hoy en día, no son aplicables en la conformación de un 'situs de enfermería' La estratificación de categorías ocupacionales es un fenómeno mucho más complejo que meramente disponer lo que un grupo de profesiones 'debería' incluir o dejar fuera, por lo que sugiero su inoperatividad en la recomposición de estructuras profesionales. Representaciones contemporáneas sobre la estratificación social en el trabajo son mejor descritas a través del análisis del funcionamiento de credenciales académicas y, sobre todo, de las relaciones sistémicas a través de las cuales las profesiones tienen influencias mutuas, tanto en sus campos de práctica como en la construcción de narrativas profesionales.
\end{abstract}

Palabras clave: Sociología de las profesiones, división del trabajo, profesiones en salud, fenómenos sociológicos, cuidado de enfermería.

\begin{abstract}
Six years ago, there appeared in this same journal a proposal from the field of academic nursing, suggesting the development or even the pre-existence of an occupational situs of nursing. By using this concept, nursing could take distance from medical dominance and establish its own occupational situs, meaning that nurses would be on top of the structure and subordinate occupations on lower levels. Although promising, this proposal did not persuade other researchers into developing a debate in related journals. This omission may be partly due to the fact that, given its empirical limitations even for the sociology of professions (where the concept originates), the concept of occupational situs has fallen into oblivion. In this article I discuss why concepts such as situs and occupational situs, besides their limited usefulness today, are no longer applicable to the conformation of a 'nursing situs.' The stratification of occupational categories is much more complex than merely deciding what a group of professions should include or not include; I thus suggest the ineffectiveness of the concept in setting
\end{abstract}

* Enfermero, PhD, Postdoctoral Research Fellow. Departamento de Sociología. Universidad de Ghent, Bélgica. Email: RicardoAlexis.AyalaValenzuela@ugent.be 
up professional structures. Contemporary representations of social stratification in different occupations are best described by analysing the role of academic credentials and, especially, the systemic relations through which professions develop mutual influence both in their practical fields and in the construction of professional narratives.

Key words: Sociology of the professions, division of labour, health occupations, sociological phenomena, nursing care.

Fecha recepción: 07/07/14 Fecha aceptación: 10/07/15

\section{INTRODUCCIÓN}

Para los investigadores familiarizados con la teoría sociológica es cautivador encontrar publicaciones que evocan una discusión sobre el funcionalismo, no sólo como un capítulo más en los manuales de sociología, sino como toda una era. Para comprender ese contexto, es necesario regresar en el tiempo: Es mediados del siglo pasado en los Estados Unidos -todo objeto y acción es analizado desde su contribución 'funcional' al orden social; se busca legitimar un modelo de sociedad en un clima de creciente progreso. La ciudad huele a industria, es la era moderna. El saber científico y tecnológico experimentan una explosión jamás vista: más y más profesiones nacen, nuevas tecnologías para el hogar debutan, en especial aparatos electrodomésticos para la cocina que vendrán a apoyar el locus de la mujer en "su" función, los teléfonos de Western Electric se propagan y, con ellos, las teorías de la comunicación; Verve y Reprise registran récords de venta de long plays de Frank Sinatra y Ella Fitzgerald, el diario en blanco y negro anuncia el fin de la guerra, nace la píldora anticonceptiva. ¿Cómo podría no reinar el optimismo?

Estas imágenes que nos llegan hoy a través de posters vintage son el paisaje de un clima fecundo también para la sociología, la era funcionalista, entendiblemente también para la sociología de las profesiones. Particularmente interesantes resultan en esa época la generación y comportamiento de ocupacio- nes nuevas y la vida en sociedad de quienes ejercían las profesiones. Es en este contexto que el estatus se vuelve una preocupación cada vez más recurrente en las revistas sociológicas y, por extensión, otros conceptos relacionados, como el de situs ocupacional. El concepto de situs reaparece recientemente en la literatura de enfermería (1), luego de dar inspiración a un par de generaciones de estudios sociológicos (2-4) para luego retirarse por completo. Discutir su potencial ineficacia, no obstante, es el objetivo que persigo en el presente artículo, y aunque no se trata particularmente de un trabajo sobre historia de las ideas, dedicaré especial atención a las décadas correspondientes: al emplear conceptos que surgen en la sociología de postguerra, haré uso mayoritario de literatura sociológica de la época. El artículo finaliza con una discusión sobre vías alternativas de desarrollo profesional, planteando algunas hipótesis basadas en procesos de delegación de tareas y de diferenciación de las profesiones, con énfasis en enfermería.

\section{CONCEPTO DE SITUS}

La posición en la estratificación social se interrelaciona con múltiples otros conceptos de importancia sociológica. Su centralidad es tal que siempre surge en el análisis, cualquiera sea la categoría social que se tome como punto de partida al estudiar las diferencias entre individuos. Usando las nociones de 'pertenencia a un grupo social', 'posición relativa en las jerarquías', y 'función en 
un grupo', Benoit-Smullyan (2) propone en 1944 el uso de los términos en latín situs, status y locus, respectivamente. Situs se refiere a la pertenencia a un grupo social en que los individuos comparten una característica común no jerárquica. Tal característica del situs puede ser, por ejemplo, cultural (idioma, religión, etc.), biológica (etnicidad, edad, etc.) o geográfica (barrio, región, etc.). Por su naturaleza, estas características son, en principio, equivalentes, igualitarias, por tanto reportan filiación (situs) mas no rango jerárquico. En base a esta premisa, es fundado deducir que las profesiones, dejando de lado su mayor o menor deseabilidad social, son en principio iguales, equivalentes, pues comparten la característica de contribuir de manera particular al funcionamiento de una sociedad: ej.: a la dirección administrativa de una región, al embellecimiento de la ciudad, a la expresión artística o al cuidado del enfermo. En cambio, por status se entiende el ordenamiento de individuos en una escala de rangos superior-inferior en base a atributos socialmente deseables -sea ésta una deseabilidad política, económica o de prestigio.

Puede parecer difícil escindir el análisis entre status y situs en sociedades capitalistas, en que cada grupo social (situs) es asociado tan fuertemente en el imaginario con cierta deseabilidad económica o simbólica (status), que se desarrollan tantos mecanismos para convertir filiaciones en símbolo de rango social como mecanismos de exclusión y exclusividad. Basta imaginar las diferencias de status en base a los sexos, la diferenciación entre las ocupaciones intelectuales y las aplicadas, las preferencias por practicar ciertos deportes sobre otros, o la aparente indeseabilidad de ejercer un oficio para el que se requiere menor cualificación. Cambios en el situs, de hecho, inducen mentalmente a la idea de cambios en el status: ej.: cambio de barrio, de profesión, de marcas. Como categorías analíticas, no obstante, situs y status son dos conceptos diferentes.

Un tercer tipo de posición social plantea- do por Benoit-Smullyan, el locus, se refiere a la función con la cual el individuo contribuye a un grupo organizado, e.g. jefe(a) de familia. El término locus, de hecho, es tomado del latín loco parentis ('en lugar de los padres') para dar especificidad al término 'rol', que en teoría sociológica es también ampliamente usado en el análisis de grupos no organizados, como aquéllos en base a clase social.

Situs, status y locus refieren entonces a tres tipos diferentes de posición social; su calco hispánico 'estatus', ‘sitio' y 'lugar' pueden iluminar mejor la diferencia entre ellos. Mientras que el 'estatus' representa rangos 'superiores' o 'inferiores', el 'sitio' (o área) corresponde simplemente a la naturaleza del grupo, en tanto el 'lugar' a la función dentro del grupo. Aunque los tres tienden a operar en simultaneidad, no necesariamente lo hacen, pues corresponden a fenómenos sociales diferentes; incluso diferentes formas de status no siempre se dan de manera correlacional: estatus económico o político, por ejemplo, no siempre coexiste con admiración o deferencia, pero a menudo sí correlacionan con formas evidentes o inadvertidas de imitación, en otras palabras, aspiración.

Mientras estos conceptos son interesantes per se, es su aplicación a las ocupaciones, per contra, el principal interés de este artículo, y es lo que elaboraré en la siguiente sección. Comprender la complejidad que el entrecruzamiento de estas tres dimensiones añade al análisis del trabajo en enfermería es relevante para toda discusión sobre estructuras profesionales.

\section{CONCEPTO DE SITUS OCUPACIONAL}

Como se ha clarificado en la sección anterior, existe una dimensión de verticalidad evidente entre diferentes ocupaciones, pudiendo organizarlas en una escala de deseabilidad social. El concepto de situs, en cambio, introduce una diferenciación horizontal entre ocupaciones. Hay fundadas razones para sostener que una estratificación unidimensional 
no es un modelo óptimo para comprender el funcionamiento ocupacional de una sociedad, al constituir una sobre-simplificación y, por tanto, una representación grosera, irrealista -como sostienen Morris \& Murphy (3)-, como son los escalones empleados con frecuencia en censos de población. Morris \& Murphy (4) emplean la noción de situs de Benoit-Smullyan (2) para ampliar el modelo de funcionamiento de las ocupaciones mediante "categorías de trabajo" de alguna manera diferenciadas. Usando su mismo ejemplo, vendedor y operario de manufactura son ocupaciones diferentes en situs, aun cuando esta diferenciación no tenga relación con el status, posiblemente similar, que puedan disfrutar. Al combinar las dos categorías, status y situs, resulta un modelo con rangos 'superior-inferior' (status) que cruzan diferentes estratos de ocupaciones organizadas por áreas de afinidad (situs) horizontalmente. El modelo resultante parecía prometer una representación bidimensional, característica central del modelo.

$\mathrm{Al}$ surgir en una época de gran optimismo -los escenarios científicos y productivos cobran vigor en la sociología de postguerra, con el consiguiente debate sobre qué es una profesión y qué un oficio $(6,7)$ - no es sorpresa que esta discusión en los Estados Unidos coincida con una intensa discusión en los círculos de enfermería sobre hasta qué punto esta ocupación calificaba o no como profesión $(8,9)$. Esto trae a un primer plano los mecanismos para lograr ascenso social para las enfermeras, convirtiéndose indiscutiblemente en una de las lides por prestigio más notorias y duraderas de toda la literatura sobre profesiones (10).

En este contexto de reconstrucción del mundo occidental tras la guerra, hay intentos por validar una determinada concepción de sociedad, consagrada en la vasta sociología funcionalista de Parsons (11). En ella, surgen modelos funcionalistas -aquellos que explican los grupos sociales como 'funcionales' para una sociedad que adquiere estabi- lidad- desde los cuales es posible evaluar la funcionalidad de las ocupaciones respecto a las necesidades sociales (ej.: salud) y en qué medida una ocupación aislada o bien un grupo de ocupaciones es suficiente para cubrir tales necesidades. Así, para el caso del situs salud, hay un grupo de ocupaciones actuando en interdependencia que busca cubrir las necesidades para que la sociedad tenga funcionalidad en sus otras esferas (arquetípicamente, la esfera del trabajo). Lo que es pertinente extractar de esto es que un situs es un área o categoría 'funcional' de ocupaciones respecto al orden social, que en cada situs se encuentran profesiones que lo hacen funcionar, y que estas ocupaciones pueden disfrutar diferentes rangos de status, respecto al mismo situs o respecto de otros.

Si bien este concepto fue usado hasta la década de 1970 (12, 13), la variabilidad de las categorías horizontales entre respondientes hizo caer el situs en desuso. Una ocupación, por ejemplo, la de enfermera, podría hoy calificar para el situs 'salud', pero también para el de 'administración'; u otros, como el de abogado en el situs de 'autoridad legal', pero también en el de 'educación', o el de recolector de basuras en 'mantenimiento' y también en 'salud'. Aun dejando estas ambigüedades de lado, hasta este punto de mi análisis, ni en el trabajo de Benoit-Smullyan (2) ni en los de Morris \& Murphy $(3,4,14)$ se advierte la intención de explicar mediante estos conceptos la esfera técnica de las ocupaciones, que es el uso que hacen Milos et al. (1) en su argumento, abogando por un eventual situs de enfermería. El situs ocupacional es, ante todo, un término que captura un fenómeno de diferenciación social.

Existe, no obstante, un modelo publicado en 1969 (15) en que las dimensiones horizontal y vertical (status y situs) son combinadas en una representación piramidal, asignando al ápice del situs el lugar para las 'profesiones verdaderas' -por antonomasia, la ocupación con los conocimientos totales para el funcionamiento del situs ocupacional 
completo- y posiciones más bajas a las que al no ostentar tales conocimientos recibían la denominación de 'pseudo-profesiones', 'para-profesiones' o 'semi-profesiones' $(15,16)$, y en la base de la pirámide a las ocupaciones no profesionales. Lo que esta revisión ${ }^{1}$ clarifica respecto a este modelo de situs ocupacional, a pesar de su aparente poder descriptivo sobre el surgimiento de nuevas ocupaciones en la época y sobre la coexistencia de ocupaciones interconectadas, es que su aplicación empírica se ve debilitada por la existencia de situs ocupacionales completos con mayor deseabilidad social que otros. Por ejemplo, por mucho tiempo la beneficencia social se consideraba de mayor prestigio que la educación de una nación. Así, la característica de horizontalidad y paralelismo entre diferentes situs tiende a desaparecer, y con ella la bidimensionalidad del modelo (17).

Del mismo modo, diferentes tipos de situs tienen lógicas de funcionamiento distintas y en pocos casos pueden considerarse come estructuras homólogas de distintas áreas de trabajo. Por ejemplo, dependiendo de la naturaleza objeto de trabajo del situs, la división del trabajo puede tener distintos grados de nitidez (más en construcción, menos en salud), la intercambiabilidad de roles puede tener distintos grados de tolerancia (menos en el ejército, más en salud), y las fuerzas internas de las organizaciones tener distintos

\footnotetext{
${ }^{1}$ Nota metodológica: el concepto de 'revisión' aquí adquiere una connotación diferente a la de 'revisión sistemática' empleada en la investigación en salud a partir de 'variables'. En la investigación conceptual, una revisión usualmente comienza no con términos estrictos de búsqueda (que podríamos denominar 'cerrada'), sino con 'nociones' (que por contraposición podríamos denominar 'abierta') y los contextos sociales e históricos en que éstas surgen; en el caso de interés, las profesiones en la sociología de postguerra y las aspiraciones sociales de la época. La metodología, entonces, consiste en la adopción de una actitud receptiva hacia los conceptos, lectura de publicaciones pertinentes, identificación de la afiliación epistémica de los autores, examen de los referentes empleados, rastreo de publicaciones que hacen retrorreferencia a las obras en cuestión, revisión de conceptos circundantes y de familias de conceptos, familiarización con obras paralelas de un mismo autor, lectura horizontal de las revistas de corriente principal, y un examen cruzado e interpretativo de estas fuentes en su conjunto.
}

grados de influencia (menor en el arte, mayor en salud). A través de diferentes mecanismos de orden simbólico, discursivos y no discursivos, un situs refleja finalmente una cultura de trabajo en particular.

Otro aspecto que debilita el concepto de situs ocupacional amenazando su desalojo del debate sobre profesiones es que a pesar de poner en perspectiva aspectos de importancia, como es el funcionamiento de los 'grupos ocupacionales', en el análisis de procesos de diferenciación social $(4,18,19)$ ignora la gran influencia ejercida por el comportamiento social de los individuos que practican una ocupación dada. Aun considerando la función de la ocupación, el ejercicio individual da forma a las habilidades intelectuales y manuales y a la orientación que la ocupación adopta, asignándole un potencial político que varía dentro de un mismo situs, potencial que puede operar en un determinado escenario local mas no en otros (20). Del mismo modo, grupos no profesionales, 'proletarios', a menudo ejercen estrategias basadas en movilización de masas, que en general tienden a desencadenar más repercusiones políticas (prácticas o simbólicas) que las empleadas por ocupaciones con más preparación, influyendo precisamente en el status político.

Es fundado pensar también que la noción de situs ocupacional, además de sus limitaciones empíricas, es desalojada definitivamente de la discusión sociológica en la medida que otros paradigmas desplazaron el funcionalismo de postguerra, que la sociedad y el trabajo pasan a tomar creciente complejidad y que el modelo social recibe cuestionamientos desde distintos frentes. Asimismo, si la utilidad dada al situs ocupacional fue la de predecir actitudes, comportamientos y estilos de vida en función de la ocupación de un individuo, con la consiguiente formación de subculturas $(3,4,14)$, su aplicación es sustituida más tarde por el estudio de identidad de clases.

Habiendo revisado el auge y caída del 
concepto de situs en el análisis de las ocupaciones en cuanto a sus dinámicas en la estratificación social, es necesario discutir si aún quedan posibilidades para la conformación de un eventual situs de enfermería, como ha sido propuesto (1) o si otros marcos pueden orientar nuevas vías para el desarrollo futuro de la profesión.

\section{SITUS DE ENFERMERÍA}

Como he explicado en las secciones anteriores, las categorías organizativas de las ocupaciones no son construidas de manera arbitraria, sino modeladas socialmente en base a deseabilidad social (status) y funcionalidad social (situs). Con esto en mente, parece difícil considerar el ámbito de la enfermería como un situs (1) per se, pues, según este análisis muestra, un situs ocupacional corresponde a un agrupamiento que es funcional a las necesidades sociales, no a una categoría ocupacional aislada ni a un subgrupo de categorías interrelacionadas.

Con el objeto de establecer un análisis más nítido, es necesario separar las necesidades sociales, por un lado, de las ocupaciones que satisfacen tales necesidades, por otro. Entendido de esta manera, una comunidad no experimenta necesidad de odontología o de enfermería o de dietética en sentido estricto, sino de salud. Como reflejo, las profesiones vienen a cubrir, o al menos contribuir a cubrir, tal necesidad. Las profesiones de hecho surgen en esa lógica como reflejo de necesidades sociales, sean éstas preexistentes o introducidas de manera intencionada en el público, transformando discursivamente carencias en problemas tratables (ej.: exceso de peso en obesidad, ignorancia en riesgo tributario, falta de fe en condena del alma) y brindando soluciones especializadas para esos problemas (ej.: prescripción de regímenes, asistencia contable, salvación espiritual) (21-23). Las necesidades de salud de una familia, desde la información necesaria para seleccionar alimentos e implementos de cuidado personal adecuados, hasta las intervenciones quirúrgicas más complejas son, de hecho, cubiertas por un número creciente de ocupaciones. La propuesta de desarrollar un 'situs de enfermería' (1), por lo menos en el sistema actual de jurisdicciones profesionales $(5,6,22)$, pierde entonces plausibilidad. Del mismo modo, plantear un situs de construcción urbana en que únicamente operen arquitectos o únicamente ingenieros parece también inadecuado, pues la necesidad social de obras es cubierta por una serie de ocupaciones, desde la concepción hasta la decoración, pasando por cálculos, selección de materiales y mano de obra. No obstante, en la discusión final, la utilidad del situs en el funcionamiento contemporáneo de las ocupaciones encuentra las limitaciones antedichas, y por ende no abundaré en lo infructífero de su aplicación hoy; un modelo es útil en tanto representa la realidad -de manera simplificada, pero la representa- y deja de serlo en tanto el objeto de estudio cambia, de forma que el modelo pierde su potencial, sea teórico o metodológico. De esto se desprende que su uso en el análisis de la enfermería aplicada puede conducir a la idea errática de escisión completa de la profesión dominante en salud, pues independientemente de las jerarquías socialmente construidas existe también una estratificación -ergo subordinación- de tareas.

La delegación en el trabajo que implica un situs, igualmente abordada en aquel artículo, puede ser considerada crucial en el desarrollo de distintos campos de experticia. Especialmente durante la industrialización, es precisamente la delegación de trabajo indeseado a ocupaciones subordinadas $(20,21)$ lo que da lugar a la apropiación de parte de éstas y, eventualmente, al surgimiento de un número de profesiones sin precedentes, a través de procesos de fisión, fusión y mutación, los mismos que explican parte del desarrollo técnico de la enfermería durante el siglo XX $(20,21)$. Es a través de la delegación de tareas indeseadas desde la profesión médica que la 
enfermería absorbe y apropia procedimientos y técnicas. Es intrigante hipotetizar a) si el proceso que hoy da lugar a la noción de 'gestión del cuidado' no es más que la continuación de un proceso ininterrumpido de delegación de trabajo indeseado (21), en que la denominación viene a ser el nombre 'deseable' para ese nuevo conjunto de funciones, roles $\mathrm{y}$ tareas; $\mathrm{y} b$ ) si tal proceso de delegación dará lugar a una nueva forma de enfermería técnica, encarnada por auxiliares y técnicos, que evolucionará hacia la forma de enfermería profesional, mientras que el ejercicio de las actuales enfermeras mutará, por un lado, hacia aspectos puramente gerenciales de salud, y por otro lado, hacia formas más intervencionistas del cuidado en salud. Con todo, es preciso subrayar que estas transformaciones distan de ser un fenómeno puramente técnico o aun jurídico -la evolución de las profesiones es, ante todo, un proceso de transformación social.

El planteamiento de Milos et al. (1) se refiere también a la noción de división del trabajo en su sentido menos progresista, el de planificación versus ejecución; en otras palabras, división entre trabajo intelectual y trabajo manual. Si bien ésta es una idea que el situs ocupacional recoge en su formulación original, este sentido de división del trabajo subraya, y de hecho asiente, la formación discursiva de élites profesionales $(6,7$, 21-23), ergo la noción resulta extemporánea al panorama actual de distribución de roles entre ocupaciones. Las ocupaciones técnicas, por ejemplo, no han experimentado un regreso a las tareas rudimentarias del auxiliar proto-técnico de antaño; por el contrario, reciben hoy en día educación avanzada, incluso en universidades, con componentes científicos en creciente proporción. En efecto, si fuera factible efectuar comparaciones retrospectivas, podría hipotetizar que su preparación puede en la actualidad ser superior, académica e instrumentalmente hablando, a la recibida por las profesiones universitarias de mediados del siglo XX. Resultaría in- exacto considerarlas hoy como una actividad puramente manual, pues en las dinámicas de división del trabajo de hoy las líneas divisorias nítidas entre trabajo intelectual y trabajo manual tienden a desaparecer $(5,6,19,20)$, fenómeno también ocurrido en la línea que separaba las categorías denominadas antiguamente profesiones, semi-profesiones y ocupaciones no profesionales (13). En este mismo ejemplo, cuando la literatura internacional hace referencia a la profesión de enfermera (nurse), tal denominación se extiende a todas las categorías dentro de la enfermería; esta información, relevante no sólo para cálculos de dotación de personal en una perspectiva comparativa internacional realista, ayuda también a entender procesos de redefinición de las lógicas internas de una ocupación, teniendo a la vista la idea de división del trabajo más como un término organizativo que cismático; en otras palabras, se refiere a una noción focalizada más en distribuir tareas que en separar a quienes piensan el trabajo de quienes lo ejecutan. En efecto, en las organizaciones contemporáneas pocos puestos de trabajo son diseñados sólo para pensar y dirigir -por lo general ubicados exclusivamente en las altas burocracias- y también pocos (si acaso alguno) sólo para funciones ejecutorias careciendo de un entendimiento básico sobre los principios y conceptos que gobiernan tales funciones. Esta manera de concebir la división del trabajo bien puede dar pie a mutaciones como las planteadas en las hipótesis precedentes.

La sociología de las profesiones ofrece perspectivas contemporáneas más fructíferas para la investigación de campos profesionales. Gran parte de los análisis disponibles sobre la enfermería tienden a redundar en la conformación y afianzamiento de estructuras, y por ende a atribuir el desarrollo profesional a sus dinámicas internas (21-23). Un debate reemergente es el del sistema de credenciales académicas (5) en la perspectiva de un 'mercado de credenciales', el que podría orientar un análisis sobre la distribución de 
autoridad. Asimismo, modelos dirigidos al entorno de las profesiones y a sus dinámicas de interacción $(6,7,22,23)$ no sólo han puesto fin a la aplicabilidad del concepto de situs ocupacional y al de 'profesionalización', sino que han abierto una nueva era en el estudio de las profesiones, por ejemplo una serie de trabajos recientes develando aspectos hasta ahora ignorados sobre la enfermería en Chile (24-26).

\section{CONSIDERACIONES FINALES}

En este artículo he desarrollado un argumento concerniente a la reaparición del concepto de situs ocupacional, tomando como punto de partida un artículo publicado en Ciencia y Enfermería (1). Con este fin, he presentado el contexto social y teórico en que los autores más influyentes en esta sub área de la sociología abordan el concepto. El análisis permite delinear tres conclusiones de relevancia en la discusión sobre la estructura institucional de la enfermería. En primer lugar, las limitaciones empíricas del concepto de situs ocupacional, como he mostrado, hacen pensar en una justificada obsolescencia: si bien es orientador en que las profesiones actúan en una lógica jerárquica, este modelo considera más bien aspectos estructurales, internos a una ocupación dada, ignorando el objeto del área de trabajo, la realización individual de roles, la naturaleza y distribución de las tareas, la variabilidad entre áreas funcionales que redundan en perspectivas unidimensionales (15) y, sobre todo, la relación mutua entre distintos grupos profesionales dentro de un área de trabajo dada $(6,7)$. La segunda conclusión es la inadecuación conceptual de la denominación 'situs de enfermería' respecto al sistema actual de jurisdicciones profesionales $(6,7)$, que implicaría responder a todas las necesidades de salud desde una única profesión. Por último, otros elementos presentados en la publicación que he traído a discusión, sobre división del trabajo y delegación, parecen necesitar readecuación de acuerdo a los avances en la literatura internacional y a los fenómenos evolutivos de las profesiones hacia modelos inclusivos de teoría-práctica $(5-7,22)$, los que tensionan antiguas concepciones de líneas de autoridad, de construcción de élites profesionales, de segregación social y de inequidades.

Más allá de ser meras filiaciones intelectuales o susceptibilidades metodológicas, la conceptualización sobre ocupaciones, en el análisis final, encierra consecuencias políticas tanto para la enfermería y las profesiones afines como para la sociedad en su conjunto, necesarias de repensar y validar empíricamente para imaginar vías de desarrollo para el siglo que avanza.

Agradecimientos: Hago pública mi gratitud hacia los académicos Tomás Koch (sociología), Bernardo Alarcón (ciencias jurídicas) y Sofía Norambuena (enfermería), por criticar versiones previas de este manuscrito.

\section{REFERENCIAS}

1. Milos P, Larraín A, Simonetti M. Categorización de servicios de enfermería: propuesta para asegurar una atención de calidad en tiempos de escasez de enfermeras. Cienc enferm. 2009; XV(1): 17-24.

2. Benoit-Smullyan E. Status, status types, and status interrelations. Am Sociol Rev. 1944; 9(2): 151-161.

3. Morris RT, Murphy RJ. The utility of Situs Analysis in the Study of social Structure. $48^{\text {th }}$ Annual Meeting of the American Sociological Society. New York; 1953.

4. Morris RT, Murphy RJ. The situs dimension in occupational structure. Am Sociol Rev. 1959; 24(2): 231-239.

5. Collins R, Collins R. The credential socie- 
ty: An historical sociology of education and stratification. New York: Academic Press; 1979.

6. Abbott A. The System of Professions: An Essay on the Division of Expert Labor. Chicago: The University of Chicago Press; 1988.

7. Abbott A. Chaos of Disciplines. Chicago, IL: University Chicago Press; 2001.

8. Bixler G, Bixler R. The professional status of nursing. Am J Nurs. 1945; 45(9): 730735.

9. Bixler G, Bixler R. The professional status of nursing. Am J Nurs. 1959; 59(8): 11421147.

10. Cutcliffe JR, Wieck K. Salvation or damnation: Deconstructing nursing's aspirations to professional status. J Nurs Manag. 2008; 16(5): 499-507.

11. Parsons T. Essays in sociological theory. New York: Simon and Schuster; 2010.

12. Pavalko R. Sociology of Work and Occupations. Itasca, Illinois: Peacock; 1971.

13. Dunkerley D. Occupations and society. New York: Routledge; 2013.

14. Morris RT, Murphy RJ. Occupational situs, subjective class identification, and political affiliation. Am Sociol Rev. 1961; 26(3) 383-392.

15. Goode JW. The Theoretical Limits of Professionalization. In Etzioni A. The semi-professions and their organization. Teachers, Nurses, Social Workers. New York: The Free Press; 1969. p. 266-313.

16. Etzioni A. The semi-professions and their organization. Teachers, Nurses, Social Workers. New York: The Free Press; 1969.
17. Gerstl JE, Cohen LK. Dissensus, situs and egocentrism in occupational ranking. Brit J Sociol. 1964; 15(3): 254-261.

18. Hatt PK. Occupations and Social Stratification. Am J Sociol. 1950; 45: 533-543.

19. Foote N, Hatt PK. Social Mobility and Economic Advancement. Am Econ Rev. 1953; 43: 371-373.

20. Hall NE, Schwirian KP. Occupational situs, community structure, and local political participation. Sociol Focus. 1968; 1 (3): 17-30.

21. Dingwall R. Essays on Professions. Aldershot: Ashgate; 2008.

22. Abbott A. Varieties of ignorance. Am Soc. 2010; 41(2): 174-189.

23. Evetts J. New directions in state and international professional occupations: discretionary decision-making and acquired regulation. Work Employ Soc. 2002; 16(2): 341-353.

24. Ayala RA, Fealy GM, Vanderstraeten R, Bracke P. Academisation of nursing: An ethnography of social transformations in Chile. Int J Nurs Stud. 2014; 51(4), 603611.

25. Ayala RA, Binfa L, Vanderstraeten, R, Bracke P. Exploring sociohistorical conflicts between midwives and nurses: A perspective from Chile. J Interprof Care. 2015; 29(3): 216-22.

26. Ayala RA, Holmqvist MT, Messing HB, Browne RF. Blessed art thou among women: male nursing students and gender inequalities in Chile. Nurse Educ Today. 2014; 34(12): 1480-4. 